\title{
Dynamic game task of executors incentives in projects for the development of new production in continuous time
}

\author{
O V Pavlov ${ }^{1}$ \\ ${ }^{1}$ Samara National Research University, Moskovskoye shosse, 34, Samara, Russia, 443086 \\ e-mail:pavlov@ssau.ru
}

\begin{abstract}
The article explores the incentive problem of executors of the new products development project at the industrial enterprise in continuous time. In the process of developing new products, the learning curve effect manifests itself, which leads to a reduction in labor intensity, depending on the cumulative volume of production. The project for the new products development is considered as a managed hierarchical dynamic system, consisting of a project management board (principal) and executors (agents). The interaction of project participants is formalized as a hierarchical differential game. To solve the formulated dynamic problem of material incentives, the well-known principle of cost compensation was applied. The original problem is divided into the task of coordinated incentives and the task of coordinated planning. The study showed that the task of coordinated dynamic planning is for the principal to determine the optimal planned production volumes in order to minimize the labor cost of agents. The initial dynamic problem of material incentives was reduced to the optimal control problem. The problem of optimal control with continuous time was solved analytically using the Pontryagin maximum principle. The study identifies a condition to determine the optimal production volumes for coordination of the interests of the principal and agents.
\end{abstract}

\section{Introduction}

The article explores the incentive problem of executors of the new products development project at the industrial enterprise in continuous time. In the process of developing new products, the learning curve effect manifests itself, which means that labor time (labor intensity) is reduced to perform repetitive manufacturing operations. The project for the development of new products is considered as a managed hierarchical dynamic system consisting of the project management (principal) and executors (agents). The dynamics of a controlled dynamic production system depends only on the actions of the agents, and the principal affects the target function of the agents by choosing the material incentive function. The state of the hierarchical dynamic system in each time period depends on its position and the actions of the participants in the previous period. Production activity in the project for the development of new production is characterized by the diverging interests of the principal and agents, which leads to a decrease in the economic efficiency of the entire production system. To find a solution of these contradictions is possible by coordinating management mechanisms that encourage agents to choose actions that are beneficial to the principal. 
Dynamic models of the interaction of unequal players are considered in the active systems theory [1-2], hierarchical information systems theory [3-5] and dynamic games theory [6-8].

The dynamic incentives task of agents in terms of the dynamic games theory is called the inverse Stackelberg game. A review of reverse Stakelberg games models has been carried out in scientific publications [9-12]. In the hierarchical information systems theory [3-5] the dynamic incentives task was called the Germeier's game $\Gamma_{2}$.

The theory of active systems [1] develops the approach based on the principle of cost compensation. The principal compensates the agent's costs in the case of choosing the optimal planned trajectory and does not pay material compensation in other cases. The original problem is divided into the task of coordinated incentives and the task of coordinated planning. The task of coordinated planning is reduced to the problem of optimal control. The recent study [13] explores the results that generalize the theorems in the monograph [1].

The hierarchical systems theory [3-5] suggests the approach that used the choice of the principal of the program of joint actions with the agent and punishment for deviation from this program. As a result, the initial problem is transformed into the optimization problem.

In the dynamic games theory [7], the principal plan is implemented using trigger strategies. The basic idea is that agents agree to follow a certain trajectory and punish any deviated agent.

The current study formulates and analytically solves the dynamic incentives task of agents in the conditions of learning-by-doing within the framework of the approach proposed in the monograph [1].

\section{Dynamic game task of executors incentives in projects for the development of new production}

\subsection{The general statement and decision algorithm of a task of executors incentives in projects for the} development of new production

In this dynamic game model there are dynamics of decision making and dynamics of the managed system. The inequality of participants is fixed by the moves order, the first move is made by the principal. It is assumed that agents are not linked to each other and perform actions independently.

The incentive problem is formalized dynamic game in positional strategies for two players with feedback on management:

$$
\begin{gathered}
J_{p}=\int_{0}^{T} e^{-\delta t}\{p u(t)-\sigma(x(t)\} d t \rightarrow \text { max }, \\
J_{a}=\int_{0}^{T} e^{-\rho t}\{\sigma(x(t))-C(x(t), u(t))\} d t \rightarrow \text { max }, \\
\frac{d x(t)}{d t}=u(t), \\
0<u(t) \leq x_{0}+R-x(t), t=0, T, \\
x(0)=x_{0}, \\
x(T)=x_{0}+R .
\end{gathered}
$$

where $J_{p}$ is the decision making criteria of principal, $J_{a}$ is the decision making criteria of agent, $\delta$ is principal discount rate, $u(t)$ is production volume of agent at time point $t, p$ is product price, $\sigma(x(t))$ is incentive function of principal, $x(t)$ is the cumulative production volume, $T$ is the project's planning horizon, $\rho$ is agent discount rate, $C(x(t), u(t))$ is the function of the agent's labor costs in the production of products (costs at time point $t$ ),$x_{0}$ is the production volume produced by the agent before starting the project, $R$ is the production volume to be produced by the time point $T$.

The function of the agent's labor costs in production (costs at time point $t$ ) in monetary terms is defined as the product of labor intensity $c(x(t))$, production volume $u(t)$ and the cost of one hour rate $s$ :

$$
C(x(t), u(t))=s c(x(t)) u(t) .
$$


The change dynamics in labor intensity of products from the cumulative production volume is described by different models of the learning curve. The most typical models are power, exponential and logistic ones, which are described in the scientific literature [14-17].

The degree model of the learning curve has the following form:

$$
c(x(t))=a x(t)^{-b} \text {. }
$$

where $a$ are costs of the first product production, $b$ is learning index.

The learning index characterizes the speed of decrease in the unit costs of product with an increase in the cumulative production volume.

Exponential model of the learning curve:

$$
c(x(t))=k+\beta e^{-\alpha x(t)} .
$$

where $\alpha$ is learning index $k, \beta$ are parameters of the exponential model.

Logistic model of the learning curve:

$$
c(x(t))=c_{\text {min }}+\left(c_{\max }-c_{\min }\right)\left[\frac{1}{1+\beta e^{\alpha x(t)}}\right],
$$

where $c_{\min }, c_{\max }$ are minimum and maximum values of unit costs in product manufacturing, $\alpha$ is learning index, $\beta$ is logistic model parameter.

To solve the formulated problem of incentives, the principle of cost compensation is applied [1].

In accordance with the principle of cost compensation, it is enough for the principal to compensate the agent costs to encourage it to choose a planned trajectory:

$$
\sigma(x(t))=C(x(t), u(t)) .
$$

Taking into account (3) and (1), the goal function of the principal is written:

$$
J_{p}=\int_{0}^{T} e^{-\delta t}\{[p-s c(x(t))] u(t)\} d t \rightarrow \text { max. }
$$

Given that the price of part $\mathrm{p}$ is a constant value, the maximization of the integral income of the principal can be replaced by minimizing the integral labor costs of the agent:

$$
J_{p}=\int_{0}^{T} e^{-\delta t} C(x(t), u(t)) d t \rightarrow \min .
$$

The solution algorithm consists of dividing the original problem into the task of coordinated incentives and the task of coordinated planning.

1. The task of coordinated dynamic incentives.

The principal chooses a compensatory incentive system, which consists of compensating the agent's costs in case that the principal's optimal planned trajectory is chosen and there are no material payments otherwise:

$$
\sigma(x(t))= \begin{cases}C(x(t), u(t)), & \text { если } x(t)=x^{R}(t), \text { для } \forall t \in[0, T] \\ 0, & \text { если } x(t) \neq x^{R}(t), \text { для } \forall t \in[0, T] .\end{cases}
$$

2. The task of coordinated dynamic planning.

The optimal planned principal trajectory is determined from the solution of the optimal control problem:

$$
\begin{gathered}
J_{p}=\int_{0}^{T} e^{-\delta t} C(x(t), u(t)) d t \rightarrow \min . \\
\frac{d x(t)}{d t}=u(t), \\
0<u(t) \leq x_{0}+R-x(t), \quad t=0, T, \\
x(0)=x_{0},
\end{gathered}
$$




$$
x(T)=x_{0}+R .
$$

The task of the principal is to select the optimal production volumes of parts $u(t)^{o p t}$ satisfying the constraint (6), which transfer the production process (5) from the initial state (7) to the final state (8) and minimize the integrated discounted labor costs of the agent (4).

\subsection{Solution of the dynamic production planning problem}

To solve the formulated optimal control problem with continuous time (4)-(8) we apply the Pontryagin maximum principle [18]. The direct application of the Pontryagin maximum principle to the formulated optimal control problem is impossible, since in this case there is a special control [19].

As the principal's optimality criterion, we consider the criteria of minimizing the integral discounted rate of the labor cost function of agent $C(t)$, which is close in economic terms:

$$
J_{p}=\int_{0}^{T} e^{-\delta t} \frac{\dot{C}(t)}{C(t)} \mathrm{d} t \rightarrow \min .
$$

where $\frac{\dot{C}(t)}{C(t)}=[\ln C(t)]^{\prime}$ is the logarithmic derivative of the labor cost function, which has the economic meaning of the rate of labor cost function.

\section{Statement 1}

For a positive and absolutely continuous function $C(t)$, the maximization (minimization) of the following functional

$$
\tilde{J}=\int_{0}^{T} e^{-\delta t} \frac{\dot{C}(t)}{C(t)} \mathrm{d} t
$$

is equivalent to the functional maximizing (minimizing):

$$
J=\int_{0}^{T} e^{-\delta t} \ln C(t) \mathrm{d} t .
$$

The proof of the statement is given in the Appendix.

Taking into account this statement, we take minimization of the total discounted logarithmic function of labor costs as the criteria of optimality (10). We substitute the expression for the labor cost function (1) into the functional (10):

$$
J_{p}=\int_{0}^{T} e^{-\delta t} \ln [s c(x(t)) u(t)] \mathrm{d} t
$$

To solve the formulated optimal control problem (5)-(8), (11), we apply the Pontryagin maximum principle [18]. Hamiltonian function is stated below:

$$
H(t, x, \psi, u)=\psi(t) u(t)-e^{-\delta t} s-e^{-\delta t} \ln [c(x(t))]-e^{-\delta t} \ln [u(t)],
$$

where $\psi(t)$ is an auxiliary variable that satisfies the following conjugate equation:

$$
\frac{\mathrm{d} \psi}{\mathrm{d} t}=-\frac{\partial H}{\partial x}=e^{-\delta t} \frac{\partial\{\ln [c(x(t))]\}}{\partial x} .
$$

In accordance with the Pontryagin maximum principle, at each point of the optimal trajectory the Hamiltonian function reaches its maximum with respect to the control parameters. The maximum of the control Hamiltonian is found from the condition:

$$
\frac{\partial H}{\partial u}=0
$$

We define the optimal control from the condition (12):

$$
u(t)^{o p t}=\frac{e^{-\delta t}}{\psi} .
$$

The system of conjugate equations can be written as follows: 


$$
\left\{\begin{array}{l}
\frac{\mathrm{d} x}{\mathrm{~d} t}=\frac{e^{-\delta t}}{\psi} \\
\frac{\mathrm{d} \psi}{\mathrm{d} t}=e^{-\delta t} \frac{\partial\{\ln [c(x(t))]\}}{\partial x}
\end{array}\right.
$$

From the equations of system (14) it follows:

$$
\begin{gathered}
\mathrm{d} t=e^{\delta t} \psi d x \\
\mathrm{~d} t=e^{\delta t}\left(\frac{\partial\{\ln [c(x(t))]\}}{\partial x}\right)^{-1} \mathrm{~d} \psi
\end{gathered}
$$

The symmetric form of the system (14) taking into account equations (15), (16) will have the form:

$$
\mathrm{d} t=\psi d x=\left(\frac{\partial\{\ln [c(x(t))]\}}{\partial x}\right)^{-1} \mathrm{~d} \psi .
$$

Using the separation of variables in the second differential equation (17):

$$
\frac{\mathrm{d} \psi}{\psi}=\frac{\partial\{\ln [c(x(t))]\}}{\partial x} \mathrm{~d} x .
$$

Find the general solution to the differential equation (18):

$$
\psi=C_{0} c(x(t)) \text {. }
$$

where $C_{0}$ is the integration constant.

The optimal control (13) taking into account (19) takes the following form:

$$
u(t)^{o p t}=\frac{e^{-\delta t}}{C_{0} c(x(t))} .
$$

Based on the obtained condition for optimal control (20), we formulate the following statement.

\section{Statement 2.}

Taking into account the discounting, the optimal production volumes for any model of the learning curve at each time point should be inversely proportional to the labor intensity of the products and directly proportional to the discount rate.

In the case of absence of discounting (discount rate $\delta=0$ ), the optimal control will be written:

$$
u(t)^{o p t}=\frac{1}{C_{0} c(x(t))} .
$$

Based on the obtained conditions for optimal control without discounting, we formulate the following statement.

\section{Statement 3.}

In the case of no discounting, the optimal production volumes for any model of the learning curve at each time point should be inversely proportional to the labor intensity of the products.

Find the optimal control and optimal trajectory for the power model of the learning curve (2). The formula (2) can be substituted in the resulting expression for the conjugate variable (19):

$$
\psi=C_{1} x(t)^{-b} .
$$

where $C_{1}=C_{0} a$ is the integration constant.

We substitute formula (21) into the differential equation (15):

$$
\mathrm{d} t=e^{\delta t} C_{1} x^{-b} d x .
$$

The general solution to equation (22) will have the form:

$$
t=-\frac{1}{\delta} \ln \left[C_{2}-C_{1} \delta \frac{x^{1-b}}{1-b}\right]
$$

We define the integration constants $C_{1}$ and $C_{2}$ from the boundary conditions (7) and (8): 


$$
\begin{gathered}
C_{1}=\frac{\left(1-e^{-\delta T}\right)(1-b)}{\delta\left[\left(x_{0}+R\right)^{1-b}-x_{0}^{1-b}\right]} ; \\
C_{2}=1+\frac{\left(1-e^{-\delta T}\right) x_{0}^{1-b}}{\left(x_{0}+R\right)^{1-b}-x_{0}^{1-b}} .
\end{gathered}
$$

Substituting the constants of integration (24), (25) into formula (23), we find the equation of the optimal trajectory of the cumulative production volume:

$$
x(t)^{o p t}=\left(x_{0}^{1-b}+\frac{\left(1-e^{-\delta t}\right)}{\left(1-e^{-\delta T}\right)}\left[\left(x_{0}+R\right)^{1-b}-x_{0}^{1-b}\right]\right)^{\frac{1}{1-b}} .
$$

We define the optimal control by substituting the formula (21) into the condition (13) with the found expression for $C_{1}(24)$ :

$$
u(t)^{o p t}=\frac{\delta e^{-\delta t}}{1-e^{-\delta T}}\left(x_{0}^{1-b}+\frac{\left(1-e^{-\delta t}\right)}{\left(1-e^{-\delta T}\right)}\left[\left(x_{0}+R\right)^{1-b}-x_{0}^{1-b}\right]\right)^{\frac{b}{1-b}} \frac{\left(x_{0}+R\right)^{1-b}-x_{0}^{1-b}}{(1-b)} .
$$

Find the labor cost function (1), taking into account formulas (26) and (27) on the optimal trajectory with optimal control:

$$
C\left(t, x^{o p t}, u^{o p t}\right)=a \frac{\delta e^{-\delta t}}{1-e^{-\delta T}} \frac{\left(x_{0}+R\right)^{1-b}-x_{0}^{1-b}}{(1-b)} .
$$

Analyzing (28) we come to the conclusion that under optimal control, the change in the instant costs function depends only on the discount factor $e^{-\delta t}$.

\section{Conclusion}

The paper explores the dynamic game task of executor's incentives in projects for the development of new production in continuous time.

To solve the formulated problem of incentives, the principle of cost compensation was applied. The original task is divided into the task of coordinated incentives and the task of coordinated planning. The task of coordinated incentives is as follows. The principal chooses a compensatory incentive system, which consists of compensating the agent's expenses in case of the principal chooses an optimal trajectory, or there are no material payments otherwise.

As a result of the study, a condition for the optimal production volumes determining coordination of the interests of the principal and agents was found: the optimal production volumes for any model of the learning curve at each time point should be chosen inversely to the labor intensity of the product and directly to the discount rate. In the case of absence of discounting: the optimal production volumes for any model of the learning curve at each time point should be chosen inversely to the labor intensity of the products.

As a result of analytical problem solving for power model of the learning curve, the following formula were obtained: formula for optimal production volumes at each time point, optimal trajectory for cumulative production volumes, and formula for agent labor costs at each time point for optimal trajectory with optimal control.

\section{Appendix}

Proof of the statement.

We integrate the functional (9) by parts:

$$
\int_{0}^{T} e^{-\delta t} \frac{\dot{C}(t)}{C(t)} \mathrm{d} t=e^{-\delta T} \ln C(T)-\ln C(0)+\delta \int_{0}^{T} e^{-\delta t} \ln C(t) \mathrm{d} t .
$$

We introduce the function $g(t)$ :

$$
g(t)=e^{-\delta t} \ln C(t)
$$


Then values of the function at the initial and final moment of time are $g(0)=\ln C(0)$ and $g(T)=e^{-\delta T} \ln C(T)$. Expression (29) takes the form:

$$
\int_{0}^{T} e^{-\delta t} \frac{\dot{C}(t)}{C(t)} \mathrm{d} t=g(T)-g(0)+\delta \int_{0}^{T} g(t) \mathrm{d} t
$$

Case A. Increasing function $g(t)$.

The geometric interpretation of the integral $S_{g}=\int_{0}^{T} g(t) \mathrm{d} t$ is the area of the curvilinear trapezium, bounded above by the positive function $g(t)$, below by the axis of abscissas and by the straight lines $t=0$ and $t=T$. The rectangle area bounded above by the straight line $g(t)=g(T)$, below by the axis of abscissas and by the straight lines $t=0$ and $t=T$ can be defined on the one hand through the integral and on the other hand as the multiplication of length by height:

$$
S_{T}=\int_{0}^{T} g(T) \mathrm{d} t=T g(T) .
$$

Similarly, the rectangle area bounded above by the line $g(t)=g(0)$, below by the axis of abscissas and by the straight lines $t=0$ and $t=T$ can be found:

$$
S_{0}=\int_{0}^{T} g(0) \mathrm{d} t=T g(0) .
$$

From the formulas (31) and (32) follows that:

$$
\begin{aligned}
& g(T)=\frac{1}{T} \int_{0}^{T} g(T) \mathrm{d} t . \\
& g(0)=\frac{1}{T} \int_{0}^{T} g(0) \mathrm{d} t .
\end{aligned}
$$

Then the functional (30), taking into account formulas (33) and (34), can be written:

$$
\int_{0}^{T} e^{-\delta t} \frac{\dot{C}(t)}{C(t)} \mathrm{d} t=g(T)-g(0)+\delta \int_{0}^{T} g(t) \mathrm{d} t=\frac{1}{T} \int_{0}^{T}[g(T)-g(0)] \mathrm{d} t+\delta \int_{0}^{T} g(t) \mathrm{d} t .
$$

The integral $\int_{0}^{T}[g(T)-g(0)] \mathrm{d} t=S_{T 0}$ defines the rectangle area, bounded above by a straight line $g(t)=g(T)$, below by a straight line $g(t)=g(0)$ and by straight lines $t=0$ and $t=T$.

The formula $\delta \int_{0}^{T} g(t) \mathrm{d} t$ geometrically can be interpreted as the area of a squeezed curvilinear trapezium $\delta S_{g}$, since $\delta<1$. In the case of an increasing function, the condition is satisfied $g(T)>g(0)$. The expression $\frac{1}{T} \int_{0}^{T}[g(T)-g(0)] \mathrm{d} t=\frac{1}{T} S_{T 0}$ is a positive value and calculates the area of the squared rectangle $S_{T 0}$.

The sum of the areas of the transformed curvilinear trapezium $\delta S_{g}$ and the rectangle $\frac{1}{T} S_{T 0}$ can be defined as the area of the curvilinear trapezium, bounded above by the positive function $\lambda_{1} g(t)\left(\lambda_{1}\right.$ is the constant factor), below the axis of abscissas and the straight lines $t=0$ and $t=T$ :

$$
\int_{0}^{T} e^{-\delta t} \frac{\dot{C}(t)}{C(t)} \mathrm{d} t=\frac{1}{T} \int_{0}^{T}[g(T)-g(0)] \mathrm{d} t+\delta \int_{0}^{T} g(t) \mathrm{d} t=\int_{0}^{T} \lambda_{1} g(t) \mathrm{d} t
$$


Since $\lambda_{1}$ is a constant factor, the maximization of the functional $\int_{0}^{T} \lambda_{1} g(t) \mathrm{d} t$ will be equivalent to maximizing of the functional $\int_{0}^{T} g(t) \mathrm{d} t=\int_{0}^{T} e^{-\delta t} \ln C(t) \mathrm{d} t$. Thus, the statement is proved.

\section{Case B. Decreasing function $g(t)$.}

In the case of a decreasing function, the condition is satisfied $g(T)<g(0)$. The formula (35) will have the form:

$$
\int_{0}^{T} e^{-\delta t} \frac{\dot{C}(t)}{C(t)} \mathrm{d} t=-\frac{1}{T} \int_{0}^{T}[g(0)-g(T)] \mathrm{d} t+\delta \int_{0}^{T} g(t) \mathrm{d} t
$$

The integral $\int_{0}^{T}[g(0)-g(T)] \mathrm{d} t=S_{0 T}$ defines the rectangle area, bounded above by a straight line $g(t)=g(0)$, below by a straight line $g(t)=g(T)$ and by straight lines $t=0$ and $t=T$.

The expression $\frac{1}{T} \int_{0}^{T}[g(0)-g(T)] \mathrm{d} t=\frac{1}{T} S_{0 T}$ is a positive value and calculates the area of the squared rectangle $S_{0 T}$.

Option 1: the conditions are met $\delta>\frac{1}{T}, \delta g(T)>\frac{1}{T} g(0)$.

In this case, the difference of the areas of the transformed curvilinear trapezium $\delta S_{g}$ and the rectangle $\frac{1}{T} S_{T 0}$ can be defined as the area of the curvilinear trapezium, bounded above by the positive function $\lambda_{2} g(t)$ ( $\lambda_{2}$ is the constant factor), below by the axis of abscissas and the straight lines $t=0$ and $t=T$ :

$$
\int_{0}^{T} e^{-\delta t} \frac{\dot{C}(t)}{C(t)} \mathrm{d} t=-\frac{1}{T} \int_{0}^{T}[g(0)-g(T)] \mathrm{d} t+\delta \int_{0}^{T} g(t) \mathrm{d} t=\int_{0}^{T} \lambda_{2} g(t) \mathrm{d} t
$$

Minimization of the functional $\int_{0}^{T} \lambda_{2} g(t) \mathrm{d} t$ will be equivalent to minimizing of the functional $\int_{0}^{T} g(t) \mathrm{d} t=\int_{0}^{T} e^{-\delta t} \ln C(t) \mathrm{d} t$. The statement is proved.

Option 2: the conditions are met $\delta<\frac{1}{T}, \delta g(T)<\frac{1}{T} g(0)$.

In this case, the difference of the areas of the transformed curvilinear trapezium $\delta S_{g}$ and the rectangle $\frac{1}{T} S_{0 T}$ can be defined as the area of the inverted curvilinear trapezium, bounded above by the straight line $g(t)=\frac{1}{T} g(0)$, below by the function $\delta g(t)$ and the straight lines $t=0$ and $t=T$. The negative difference of areas can be calculated $-\int_{0}^{T}\left[\frac{1}{T} g(0)-\delta g(t)\right] \mathrm{d} t=\int_{0}^{T} \delta g(t) \mathrm{d} t-g(0)$.

Since $g(0)=$ const the minimization of this expression will be equivalent to the minimization of the functional $\int_{0}^{T} g(t) \mathrm{d} t=\int_{0}^{T} e^{-\delta t} \ln C(t) \mathrm{d} t$. The statement is proved.

Option 3: the conditions are met $\delta>\frac{1}{T}, \delta g(T)<\frac{1}{T} g(0)$. 
In this case, the difference of the areas of the transformed curvilinear trapezium $\delta S_{g}$ and the rectangle $\frac{1}{T} S_{0 T}$ can be defined as the difference of the areas of two curvilinear triangles.

The area of the first curvilinear triangle is bounded above by the function $\delta g(t)$, below by the straight line $g(t)=\frac{1}{T} g(0)$ and by the straight lines $t=0$ and $t=\tau$ (abscissa of the intersection point of the function $\delta g(t)$ and the straight line $\left.g(t)=\frac{1}{T} g(0)\right)$. The area of the second curvilinear triangle is bounded above by a straight line $g(t)=\frac{1}{T} g(0)$, below by the function $\delta g(t)$ and by straight lines $t=\tau$ and $t=T$.

The areas difference can be calculated: $\int_{0}^{\tau}\left[\delta g(t)-\frac{1}{T} g(0)\right] \mathrm{d} t-\int_{\tau}^{T}\left[\frac{1}{T} g(0)-\delta g(t)\right] \mathrm{d} t=\int_{0}^{T} \delta g(t) \mathrm{d} t-g(0)$. Since $g(0)=$ const, the minimization of this expression will be equivalent to the minimization of the functional $\int_{0}^{T} g(t) \mathrm{d} t=\int_{0}^{T} e^{-\delta t} \ln C(t) \mathrm{d} t$. The statement is proved.

\section{References}

[1] Novikov D A, Smirnov M I and Shokhina T E 2002 Mechanisms of Dynamic Active Systems Control (Moscow: IPU RAN) p 124

[2] Ugolnitsky G A 2016 Management of Sustainable Development of Active Systems (Rostov on Don: Publishing House of the Southern Federal University) p 940

[3] Gorelik V A, Gorelov M A and Kononenko A F 1991 Analysis of Conflict Situations in Control Systems (Moscow: Radio i svyaz) p 228

[4] Gorelik V A and Kononenko A F 1982 Game-theoretic Models of Decision Making in Ecological-economic Systems (Moscow: Radio i svyaz) p 144

[5] Gorelov M A and Kononenko A F 2015 Dynamic conflict models III. Hierarchical games Automation and Remote Control 2 89-106

[6] Basar T and Olsder G J 1999 Dynamic Noncooperative Game Theory (Philadelphia: SIAM) p 519

[7] Dockner E, Jorgensen S, Long N V and Sorger G 2000 Differential Games in Economics and Management Science (Cambridge: Cambridge University Press) p 382

[8] Li T and Sethi S P 2017 A review of dynamic Stackelberg game models Discrete and Continuous Dyn. Syst. B 22(1) 125-159

[9] Olsder G J 2009 Phenomena in inverse Stackelberg games. Part 2: dynamic problems J. Optim. Theory Appl. 143(3) 601-618

[10] Groot N, De Schutter B and Hellendoorn H 2012 Reverse Stackelberg games. Part I: basic framework Proc. of the 2012 IEEE Int. Conf. on Control Applications 421-426

[11] Groot N, De Schutter B and Hellendoorn H 2012 Reverse Stackelberg games. Part II: results and open issues Proc. of the IEEE Int. Conf. on Control Applications 427-432

[12] Groot N, Zaccour G and De Schutter B 2017 Hierarchical game theory for system-optimal control: applications of reverse Stackelberg games in regulating marketing channels and traffic routing IEEE Control Systems Magazine 37(2) 129-152

[13] Rokhlin D B and Ougolnitsky G A 2018 Stackelberg equilibrium in a dynamic stimulation model with complete information Automation and Remote Control 79(4) 701-712

[14] Wright T P 1936 Factors affecting the cost of airplanes J. of the aeronautical sciences 3(4) 122128

[15] Yelle L E 1979 The learning curve: historical review and comprehensive survey Decision Sciences 10(2) 302-328 
[16] Badiru A 1992 Computational survey of univariate and multivariate learning curve models IEEE Transactions on Engineering Management 39(2) 176-188

[17] Mohamad Y J 2011 Learning Curves: Theory, Models, and Applications (Boca Raton: CRC Press) p 476

[18] Pontryagin L S, Boltyansky V G, Gamkrelidze R V and Mishchenko E F 1983 Mathematical Theory of Optimal Processes (Moscow: Nauka) p 392

[19] Afanasyev V N 2003 Mathematical Theory of Control Systems Design (Moscow: Vysshaya shkola) p 614 